\title{
Inhibition Evaluation of 5-(4-(1H-pyrrol-1-yl)phenyl)-2-mercapto-1,3,4- oxadiazole for the Corrosion of Mild Steel in an Acidic Environment: Thermodynamic and DFT Aspects
}

\author{
A. M. Mustafaํ, F. F. Sayyid ${ }^{1}$, N. Betti², M. M. Hanoon ${ }^{1}$, A. A. Al-Amiery ${ }^{3,4}$, A. A. H. Kadhum ${ }^{4}$ M. S. \\ Takriff 4 \\ 1Production Engineering and Metallurgy, University of Technology, P.O. Box: 10001, Baghdad, Iraq. \\ ${ }^{2}$ Materials Engineering Department, University of Technology, P.O. Box: 1001, Baghdad, Iraq \\ ${ }^{3}$ Energy and renewable energies technology center, University of Technology, Baghdad, 10001 Iraq. \\ ${ }^{4}$ Department of Chemical and Process Engineering, University Kebangsaan Malaysia (UKM), P.O. Box: 43000, Bangi, \\ Selangor.
}

*Corresponding author: Ahmed Al-Amiery (dr.ahmed1975@gmail.com)

\begin{abstract}
In this investigation, an oxadiazole namely 5-(4-(1H-pyrrol-1-yl)phenyl)-2-mercapto-1,3,4-oxadiazole (PMO), was synthesized and explored as an inhibitor against the corrosion of mild steel in $1.0 \mathrm{M}$ hydrochloric acid environment at various solution Temperatures 303-333 K. gravimetric, and microscopic techniques, namely, weight loss (WL), and scanning electron microscopy (SEM), have been used to evaluate the inhibitive performance of the tested PMO. The results of the WL method displayed that the inhibition efficiency (\%IE) was found to increase with the inhibitor concentration, while it reduced with increasing temperature. Furthermore, the WL results reveal that PMO inhibits corrosion display an IE of $95 \%$ at the highest concentration of $0.005 \mathrm{M}$. The SEM images of the mild steel surface coupon after adding PMO revealed a wide coverage of PMO molecules on a mild steel surface. Hence, the high inhibiting efficiency acquired by the tested inhibitor was explained by the strong adsorption of PMO molecules on the surface of mild steel. A protective layer has been constructed and it separating the mild steel surface from the hydrochloric acid solution, and such adsorption was found to obey Langmuir adsorption isotherm. Moreover, the thermodynamic parameters suggested that the adsorption nature of PMO molecules on the coupon surface was chemo-physisorption. Quantum chemical calculations were conducted by density functional theory (DFT) which helps correlate the methodological findings with the theoretical investigations. The mechanism of PMO molecules as corrosion inhibitor for mild steel surface in the corrosive environment was also discussed.
\end{abstract}

Keywords: Mild steel, corrosion, inhibition, oxadiazol, pyrrole, Langmuir

\section{INTRODUCTION}

Corrosion damage not only results in high costs for inspection, repair, and replacement, but it also poses a public safety risk, necessitating the production of new substances that function as corrosion inhibitors, especially in corrosive environments [1]. One of the most practical methods for preventing corrosion is the use of organic molecules as corrosion inhibitors, which is becoming increasingly common. Organic inhibitors work by adsorption on the metal surface and the formation of a protective film, according to existing evidence [2,3]. Organic compounds with high electron density heteroatoms, such as phosphorus, sulfur, oxygen, and nitrogen, as well as those with multiple bonds that serve as adsorption centers, are effective corrosion inhibitors [4-7]. Oxadiazole derivatives have a unique affinity for preventing metal corrosion in acid solutions $[8,9]$. Because of their high chemical activity and low toxicity, these heteroatom-rich compounds can be considered environmentally friendly inhibitors [10]. Due to the environmental consciousness and the negative effects of certain chemicals, recent research efforts have focused on developing low-cost, environmentally-friendly corrosion inhibitors [11]. Mild steel is the world's most widely used engineering and building material. The corrosion of mild steel is a major academic and industrial problem that has gotten a lot of attention recently [12]. Because of the extensive use of mild steel, numerous studies on its corrosion resistance have been performed. The majority of well-known corrosion inhibitors are heterocyclic compounds with more 
heteroatoms in their aromatic or long carbon chain systems. $[13,14]$. It has long been understood that effective inhibitors should have a large number of -electrons and unshared electron pairs on the inhibitors' nitrogen or sulfur atoms to the d-orbital of iron. Organic molecules' adsorption properties are also influenced by their proportions, electron density at donor atoms, and the orbital character of donating electrons. [15,16]. The main adsorption centers are organic compounds with functional electronegative groups, electron in triple or conjugated double bonds, and the inclusion of aromatic rings in their structure, which are normally strong inhibitors. [17]. New corrosion inhibitors such as pyrazole, [18,19] triazole, [20,21] tetrazole, [22] imidazole, [23], and oxadiazole derivatives [24,25] have recently piqued the interest of many researchers. The presence of nitrogen and oxygen atoms in the aromatic system facilitates electrophilic attack, so oxadiazole derivatives with different substituents were chosen as corrosion inhibitors [26]. Quantum chemical calculations have proven to be an important method for analyzing and explaining a wide range of experimental findings. To compare the obtained data with the inhibition performance, semi-empirical methods of calculation were used. [27,28]. The current investigation intended to synthesize an oxadiazole derivative and investigate it performance as a corrosion inhibitor for mild steel in $1 \mathrm{M} \mathrm{HCl}$ solution utilizing weight loss and scanning electron microscopy techniques. The results were researched with different adsorption parameters. The conservative layer formed on the mild steel surface was described by SEM. Moreover, to realize the relation between the structure of PMO molecule and its inhibition performance, theoretical parameters namely energy gap $(\Delta \mathrm{E})$, highest occupied molecular orbital (HOMO), lowest unoccupied molecular orbital (LUMO), global hardness $(\eta)$, softness $(\sigma)$, absolute electronegativity $(\chi)$ and the fraction of electrons transferred $(\Delta N)$ and the dipole moments $(\mu)$ were calculated and investigated.

\section{EXPERIMENTAL SECTION}

\subsection{Materials.}

All the chemicals and solvents used were of analytical grade and utilized without further purification and were purchased from either Merck or Sigma Aldrich. Fouriertransform infrared spectroscopy (FTIR) spectrum was recorded utilizing a Shimadzu 8300 spectrometer. Nuclear magnetic resonance spectroscopy (NMR) was conducted with an AVANCE III $600 \mathrm{MHz}$ spectrometer (Bruker, Billerica, Massachusetts, United States of America), with dimethyl sulfoxide as the internal standard. The delta values were expressed in ppm.

\subsection{Synthesis of Corrosion Inhibitor.}

The synthesis of the 5-(4-(1H-pyrrol-1-yl)phenyl)-2mercapto-1,3,4-oxadiazole (PMO), was carried out through two steps. The first step was to synthesis 4-(1H-pyrrol-1yl)benzohydrazide (2) through the reaction of a condensation reaction between ethyl 4-(1H-pyrrol-1yl)benzoate (1) and access of hydrazine hydrate in absolute ethyl alcohol for $5 \mathrm{~h}$, given as Scheme 1, and recrystallized from ethyl alcohol to produce yellow crystals. Yield 61, MP $181-183 \mathrm{oC}$. The second step was to the synthesis of $5-(4-(1 \mathrm{H}-$ pyrrol-1-yl)phenyl)-2-mercapto-1,3,4-oxadiazole which was achieved by the dissolved of compound (2) within access ethanolic $\mathrm{KOH}$. The resulting mixture was refluxed with carbon disulfide for 12 hours, followed by the addition of distilled water to the reaction mixture. The cooled solution was filtered and neutralized with drops of $\mathrm{HCl}$, and recrystallized from benzene to produce a yellow crystal. Yield 67, MP 208-211 oC. FT-IR (cm-1): Mercapto group at 2782 and azomethine group at 1623. Proton NMR (DMSO-d6 in ppm): 7.83-7.42 (m, aromatic proton), 6.44 (s, 1H, C-H heterocyclic ring ). Carbon NMR (DMSO-d6) 171.14, 144.09, $128.33,120.83,123.51,115.77$ and 111.03 .

\subsection{Solutions.}

The hydrochloric acid (37\% analytical grade) solution was used and diluted with distilled water. The inhibitor concentrations which used were $0.001,002,0.003,0.004$ and $0.005 \mathrm{M}$ in $1 \mathrm{M} \mathrm{HCl}$.

\subsection{Mild steel coupons.}

The composition of the mild steel coupons is as follows (weight percentage): carbon, 0.210 ; manganese 0.050 ; silicon 0.380; aluminum 0.010; sulfur 0.050; phosphorus 0.090; balance iron. Mild steel pieces were mechanically cut into 4.5 $\mathrm{cm} \times 2.5 \mathrm{~cm} \times 0.025 \mathrm{~cm}$ specimens for gravimetric techniques. The surface of the mild steel specimens was abraded with emery papers and washed with acetone and double-distilled water.

\subsection{Weight loss techniques.}

Mild steel specimens were immersed in the acid solutions for $1,3,5,10$, and $24 \mathrm{~h}$, and the inhibitor concentrations used were ranged $0.001-0.005 \mathrm{M}$ at $303 \mathrm{~K}$. After the exposure times the coupons were removed, washed with deionized water, and acetone, and then dried for 2 hours in the oven at $150 \mathrm{oC}$. All experiments were repeated three times and relative weight losses of the coupons were utilized to compute the \%IE. The experiments were repeated with various concentrations of the inhibitor at different temperatures (303, 313,323 , and $333 \mathrm{~K}$ ) for $5 \mathrm{~h}$ as immersion time.

The corrosion rate (CR) was evaluated using equation (1):

$$
C_{R}\left(\frac{m m}{\text { year }}\right)=\frac{87.6 \mathrm{~W}}{a t D}
$$

where $W$ is weight loss, $a$ is surface area of the mild steel coupon, $\mathrm{t}$ is exposure time, and D is the density of the mild steel $\left(\mathrm{g} \mathrm{cm}^{-3}\right)$.

$$
\begin{aligned}
& \% I E=\frac{w_{o}-w_{i}}{w_{o}} \times 100 \\
& \theta=\frac{w_{o}-w_{i}}{w_{o}}
\end{aligned}
$$

where $w_{o}$ and $w_{i}$ are the weight loss value in the absence and presence of the PMO. 


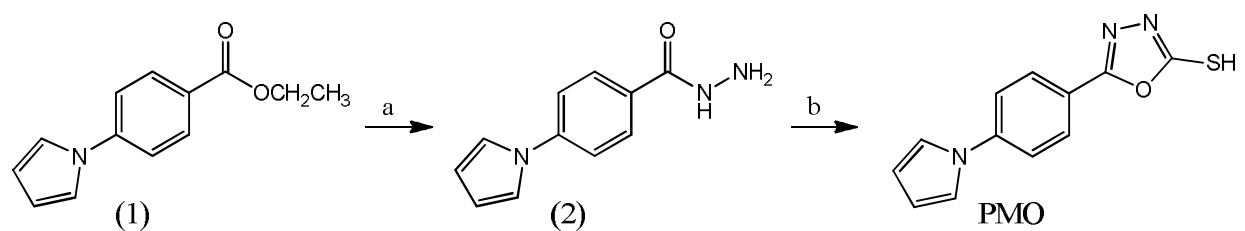

'a, hydrazine hydrate; $b$, carbon disulfide

Scheme 1. The synthesis rout of PMO

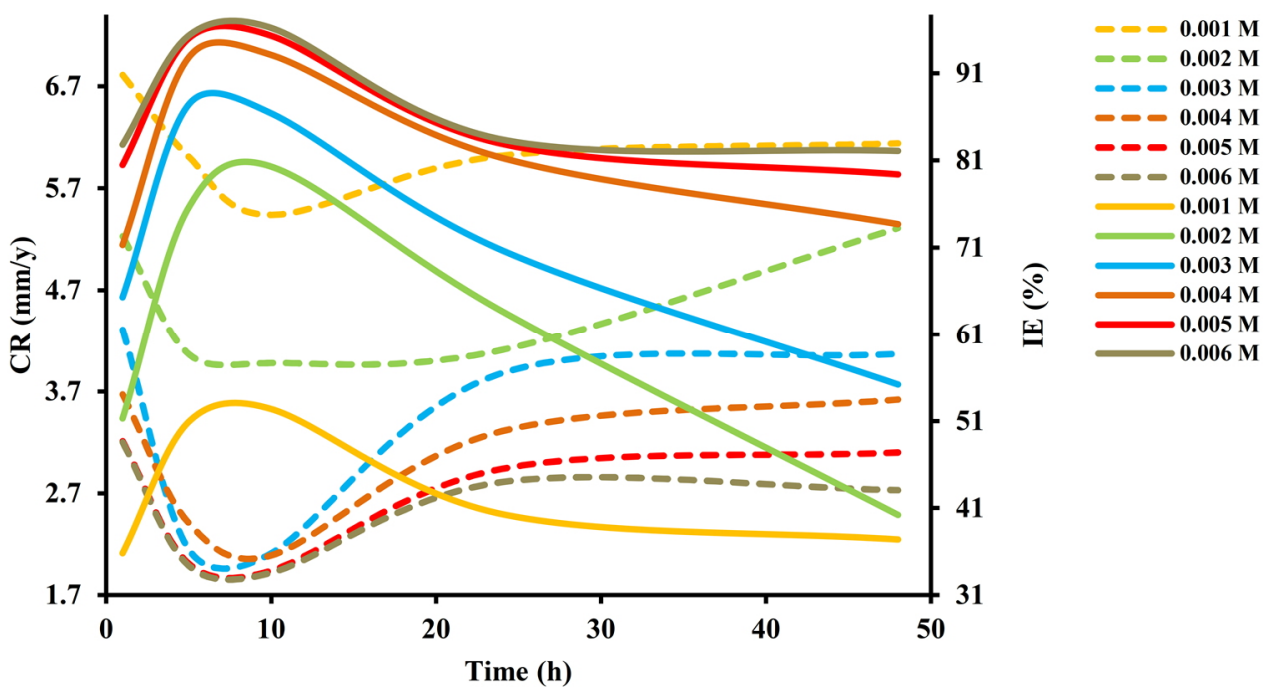

Figure 1. Corrosion rate and inhibition efficiency-time curves for the mild steel dissolution in the absence and presence of various concentrations of PMO molecules at $303 \mathrm{~K}$.

\subsection{Surface Analysis.}

The SEM analysis was achieved by using the TM1000 Hitachi Tabletop Microscope. In SEM images, the coupons were immersed in the corrosive solution in the absence and presence of $\mathrm{PMO}$ as corrosion inhibitor at the concentration of $0.005 \mathrm{M}$ and $5 \mathrm{~h}$ as immersion time.

\subsection{Quantum Chemical Calculations.}

The optimized molecular structure of the PMO molecule was geometrically by the DFT method with the $6-31+G(d, p)$ basis set. Gaussian 09 software was used. The quantum electronic parameters of the most stable conformers were calculated of the DFT. HOMO, LUMO, $\Delta \mathrm{E}, \eta, \sigma, \chi$, and $\Delta \mathrm{N}$ were calculated using equations (4-8) [29,30].

$$
\begin{aligned}
& \Delta E=E_{\text {HоMO }}-E_{\text {LUMO }} \\
& \eta=-\frac{E_{\text {HOMO }}-E_{\text {LUMO }}}{2} \\
& \sigma=\frac{1}{\eta} \\
& \chi=-\frac{E_{\text {HOMO }}+E_{L U M O}}{2} \\
& \Delta N=\frac{\chi_{F e}-\chi_{\text {inh }}}{2\left(\eta_{F e}+\eta_{i n h}\right)}
\end{aligned}
$$

where $\chi$ Fe and $\eta$ Fe were $7 \mathrm{eV} / \mathrm{mol}$ and $0 \mathrm{eV} / \mathrm{mol}$, respectively.

\section{RESULTS AND DISCUSSION}

\subsection{Synthesis of the inhibitor}

PMO has been synthesized starting from ethyl 4- $(1 \mathrm{H}-$ pyrrol-1-yl)benzoate which converts to acid hydrazide through the reaction with hydrazine. Acid hydrazide cyclized with carbon disulfide to produce PMO as a new corrosion inhibitor. Scheme 1, represents the sequence of the synthesis.

\subsection{Weight Loss Measurements.}

\subsubsection{Effect of concentration}

The mild steel corrosion rate was evaluated according to weight loss techniques in $1 \mathrm{M}$ hydrochloric acid at $303 \mathrm{~K}$ in the absence and in the presence of PMO as a corrosion inhibitor. The experimental results are demonstrated in Figure 1. From these findings, a significant reduction in weight loss was recognized with the increase in PMO concentration at $303 \mathrm{~K}$. For instance, in the $\mathrm{HCl}$ solution, without the addition of $\mathrm{PMO}$, the mild steel corrosion rate was $9.85 \mathrm{~mm} / \mathrm{y}$, which decreased to $1.94 \mathrm{~mm} / \mathrm{y}$ in presence of $0.005 \mathrm{M} \mathrm{PMO}$ at $303 \mathrm{~K}$ and $10 \mathrm{~h}$ as immersion time. The effect of PMO concentration on corrosion rate and inhibition performance of mild steel in $1 \mathrm{M}$ hydrochloric acid solution was first investigated. Figure 1 shows this activity in the presence of different $\mathrm{PMO}$ concentrations in $1 \mathrm{M}$ 
hydrochloric acid over a range of immersion times $(1,5,10$, 24 , and $48 \mathrm{~h}$ ). Figure 1 shows that as the concentration of PMO was increased, the efficiency of inhibition increased at $303 \mathrm{~K}$. As a result of this action, the amount of inhibitor adsorption and coverage on mild steel surfaces increases as the inhibitor concentration rises.

The results of Weight Loss Measurements for mild steel coupon in a hydrochloric acid environment in the presence and absence of PMO are demonstrated in Figure 1. It is an important way to improve the effect of inhibition effectiveness by increasing the concentration of the corrosion inhibitor as shown in Figure 1. The results of the tests obtained indicated that increasing the concentration of the corrosion inhibitor reduces the corrosion rate of mild steel and that the inhibition efficiency increases with increasing the concentration of the corrosion inhibitor, as shown in Figure 1. The used inhibitor PMO shows an inhibition efficiency of up to $95 \%$, at the optimum utilized concentration $(0.005 \mathrm{M})$. Moreover, the good inhibition performance is due to the bonding of molecules with the surface of the mild steel, because the inhibitor molecules have many heteroatoms such as nitrogen, oxygen and sulfur. Moreover, the higher efficiency of the PMO molecules is attributed to the presence of aromatic rings, and the resonance effect. In a corrosion inhibitor molecule, the presence of a phenyl ring and a pyrrole ring increases the electron density in the active centers, which facilitates the interaction of this inhibitor with the surface of the mild steel. The weight loss of mild steel coupons was evaluated after various immersion times $(1,5,10,24$, and $48 \mathrm{~h})$ at $(303 \mathrm{~K})$ the results are shown in Figure1. The experimental findings revealed that the presence of PMO suppressed the corrosion rate of the mild steel coupons in $1 \mathrm{M}$ hydrochloric acid environment. The inhibition efficiency was noted to increase when increasing the PMO concentration as demonstrated in Figure 1, and decrease after $24 \mathrm{~h}$, of exposure time. This can be attributed to the adsorption of PMO molecules on the surface of mild steel and after $24 \mathrm{~h}$, the PMO molecules were desorption from the steel surface. The adsorption reduces the mild steel dissolution by blocking the sites of corrosion and hence reducing the weight loss while increasing the inhibition efficiency as the concentration of the PMO increases.

\subsubsection{Effect of Time}

Time is a significant parameter when describing the corrosion inhibitor. To evaluate the stability of the adsorption rate of the tested inhibitor molecules, exposure time investigations were conducted in a $1 \mathrm{M}$ hydrochloric acid solution. The immersion time range was $1,5,10,24$, and $48 \mathrm{~h}$. The results demonstrated in Figure 1 indicate the influence of exposure time on the inhibition efficiency of $\mathrm{PMO}$ as a new corrosion inhibitor. The highest inhibitive efficiency was achieved at 5-10 h, as the early times of exposure. This result was because of prompt adsorption of PMO molecules onto the surface of mild steel due to the highest number of inhibitor molecules that block the surface of the metal from the corrosive solution. After $24 \mathrm{~h}$ of exposure, the inhibition efficiency decreased. The decrease in inhibitive efficiency was imputed to the desorption of the PMO molecules onto the surface of mild steel. In addition, the corrosion rate was increased with immersion time increase. This fact can be explained according to adsorption theory. When PMO molecules onto the surface of mild steel were desorbed, mild steel was exposed to the hydrochloric acid solution, thereby increasing the contact of a mild steel surface/corrosive environment, which results in the dissolution of metal. Furthermore, the corrosion rate increases with increasing exposure time, which was imputed to a low number of PMO molecules in a corrosive environment to control or reduce the dissolution of metal; it is obvious that after PMO molecules were desorbed, they turned inefficient and thus did not participate in the inhibitive approach.

\subsubsection{Effect of Temperature}

Weight loss measurements of mild steel were performed in an environment of $1.0 \mathrm{M}$ of hydrochloric acid in an optimum exposure time $(5 \mathrm{~h})$ in the presence of the tested corrosion inhibitor and at concentrations of $(0.001,0.002$, $0.003,0.004$, and $0.005 \mathrm{M}$ ) at different temperatures (303-333 $\mathrm{K})$. Figure 2 shows the corrosion rate and efficiency at the immersion time $(5 \mathrm{~h})$ at different temperatures (303-333 K). The corrosion rate and inhibition efficiency values of the $\mathrm{PMO}$ as an investigated corrosion inhibitor are shown in Figure 2. From Figure 2, it is evident that at a constant temperature, the corrosion rate value was reduced while the inhibition efficiency was increased with the increase of PMO concentration. This can be attributed to the increased absorption coverage of the PMO molecules on the mild steel surface in its concentrations, which reduces the dissolution rate of mild steel. Thus, PMO is an excellent inhibitor of the corrosion of mild steel in the solution of $1.0 \mathrm{M}$ hydrochloric acid under the optimum conditions of temperature (303 K) and exposure time. On the other hand, as the temperature increased at various inhibitor concentrations, and constant time $(5 \mathrm{~h})$ the value of corrosion rate increase and the inhibition efficiency was decreased. In this context, Figure 2 illustrates the change in the corrosion rate as well as the inhibition efficiency of the PMO as a tested corrosion inhibitor with different concentrations at various temperatures. This behavior can be attributed to the acceleration of the reaction of hydrogen evolution in a hydrochloric acid environment with the increase in temperature, thus reducing the absorption of the inhibitor.

\subsection{Adsorption Isotherm}

The tested inhibitor was set to be an excellent inhibitor against the corrosion of mild steel in a $1.0 \mathrm{M}$ hydrochloric acid environment. PMO molecules have heteroatoms atoms ( $\mathrm{S}, \mathrm{O}$, and $\mathrm{N}$ ), as well as heterocyclic (pyrrole and oxadiazole) and phenyl rings, which can be absorbed onto the surface of mild steel to form a protective layer. This layer can be created by one of the adsorption modes listed below [31]: 


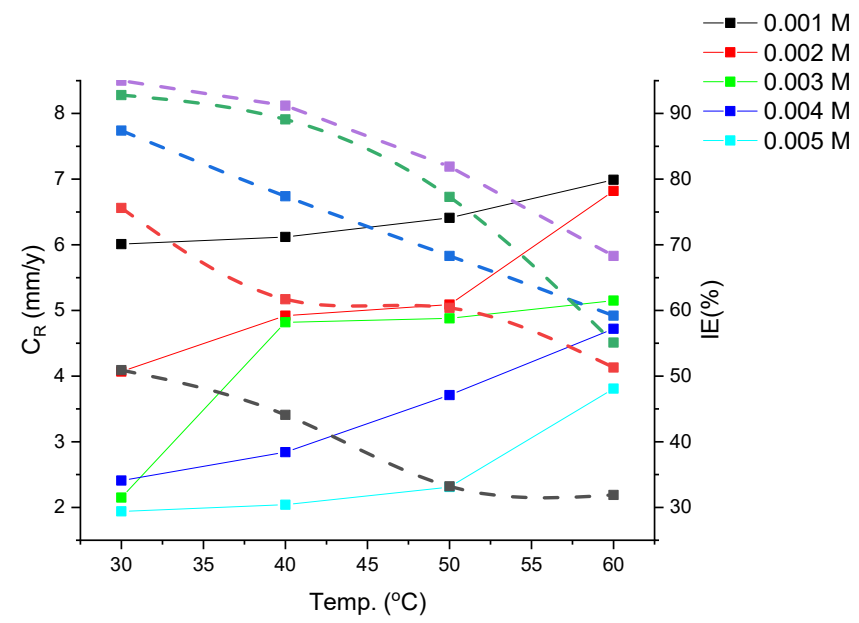

Figure 2. Corrosion rate and inhibition efficiency-Temperature curves for the mild steel dissolution in the absence and presence of various concentrations of PMO molecules at immersion time $(5 \mathrm{~h})$.

1. Physical adsorption of PMO molecules on mild steel surface due to the electrostatic attraction between the protonated sites of the PMO molecules (in the hydrochloric acid environment) and the charged surface of mild steel.

2. Chemical adsorption by forming coordination bonds between the unoccupied d-orbital of Fe atoms and the electron pairs of the sulfur, oxygen, and nitrogen atoms.

3. Coexistence of the two adsorption mechanisms.

Plots of fractional surface coverage (Cinh/) against inhibitor concentrations (Cinh) at different temperatures were used to investigate the best-fit adsorption isotherm (Langmuir, Temkin, Freundlich, or Frumkin type) of the tested inhibitor. Figure 3, shows straight lines with approximately unit slope, indicating that the studied inhibitors adsorb on the metal surface obey Langmuir adsorption isotherm as in equation (9) [32]

$$
\frac{C_{i n h}}{\theta}=\frac{1}{K_{a d s}}+C_{i n h}
$$

where Kads is the equilibrium constant.

Figure 3, demonstrates that the $K_{a d s}$ value is a high which, referring to potent adsorption of the PMO molecules on the surface of mild steel at $303 \mathrm{~K}$, on the other hand at higher temperature, the adsorbed molecules tend to desorb from the surface of mild steel. The adsorption free energy $\left(\Delta G_{\text {ads }}^{o}\right)$ was evaluated according to equation (10) [33]

$$
\left(\Delta G_{a d s}^{o}=-R T \ln \left(55.5 K_{a d s}\right)\right.
$$

The calculated value of $\Delta G_{a d s}^{o}$ for the $\mathrm{PMO}$ as an investigated inhibitor at $303 \mathrm{~K}$ is $36.87 \mathrm{~kJ} \mathrm{~mol}^{-1}$. The higher value of $\Delta G_{a d s}^{o}$ for the PMO demonstrating that the PMO molecules adsorbed potently on the coupon surface in the

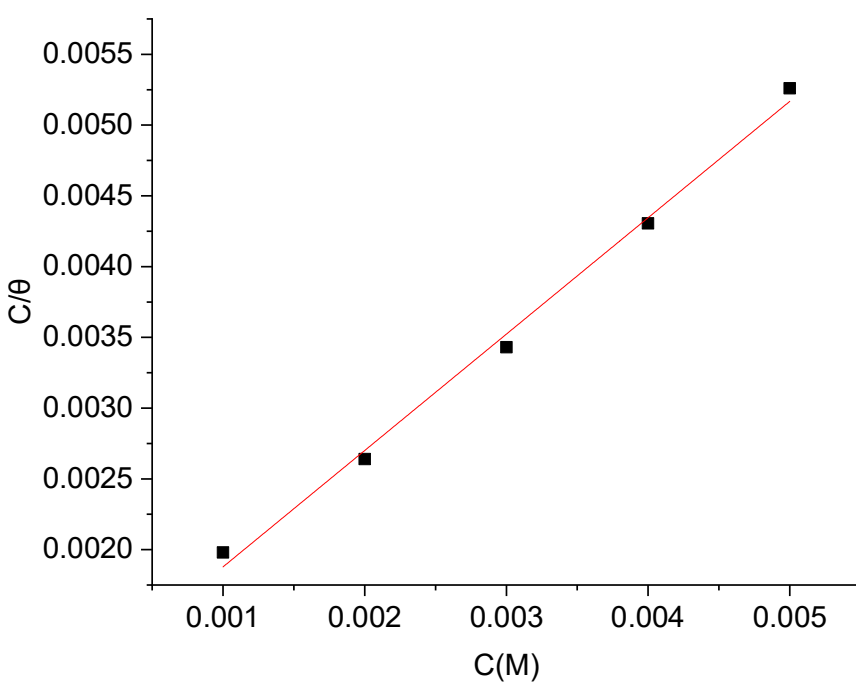

Figure 3. Langmuir adsorption isotherm for: $\mathrm{PMO}$ as corrosion inhibitor for mild steel in. 1.0 M hydrochloric acid environment

$\mathrm{HCl}$ solution. This matches the inhibition effectiveness value of the investigated corrosion inhibitor calculated using the weight loss method. The obtained $\Delta G_{a d s}^{o}$ value, on the other hand, was around $40 \mathrm{KJmol}^{-1}$, implying that the adsorption of the investigated corrosion inhibitor on the coupon surface was primarily physical [34].

\subsection{Surface Characterization}

Figure 4 shows SEM images of the mild steel surfaces coupons in 1.0 M hydrochloric acid solution free of PMO and in the presence of PMO ( $a$ and $b$ ). After a 5-hour immersion in $1.0 \mathrm{M}$ hydrochloric acid, Figure 4(a) shows a coupon of mild steel surface that has been severely damaged by immersion in hydrochloric acid solution. SEM image after adding $0.005 \mathrm{M}$ PMO to the 1.0 M hydrochloric acid solution is demonstrated in Figure 4(b).

The mild steel surface was smooth and free from pits. It can be concluded that the corrosion rate is less in the presence of PMO molecules. These findings also support the weight loss results and quantum chemical calculations.

\subsection{Theoretical calculations}

Theoretical studies are extremely useful for learning more about the phenomenon of inhibition. The calculated quantum chemical parameters EHOMO, ELUMO, $\Delta \mathrm{E}, \eta, \sigma$, $X$, and $\Delta \mathrm{N}$ as well as Mulliken charges on the atoms, are linked to an inhibitor's corrosion inhibition performance. The optimization of the studied inhibitor molecules [35,36] will reveal these parameters. These values aid in the prediction of metal-inhibitor interactions. In this study, all of the quantum parameters for PMO molecules were computed. The theoretical calculations findings gained are displayed in Table 1. 

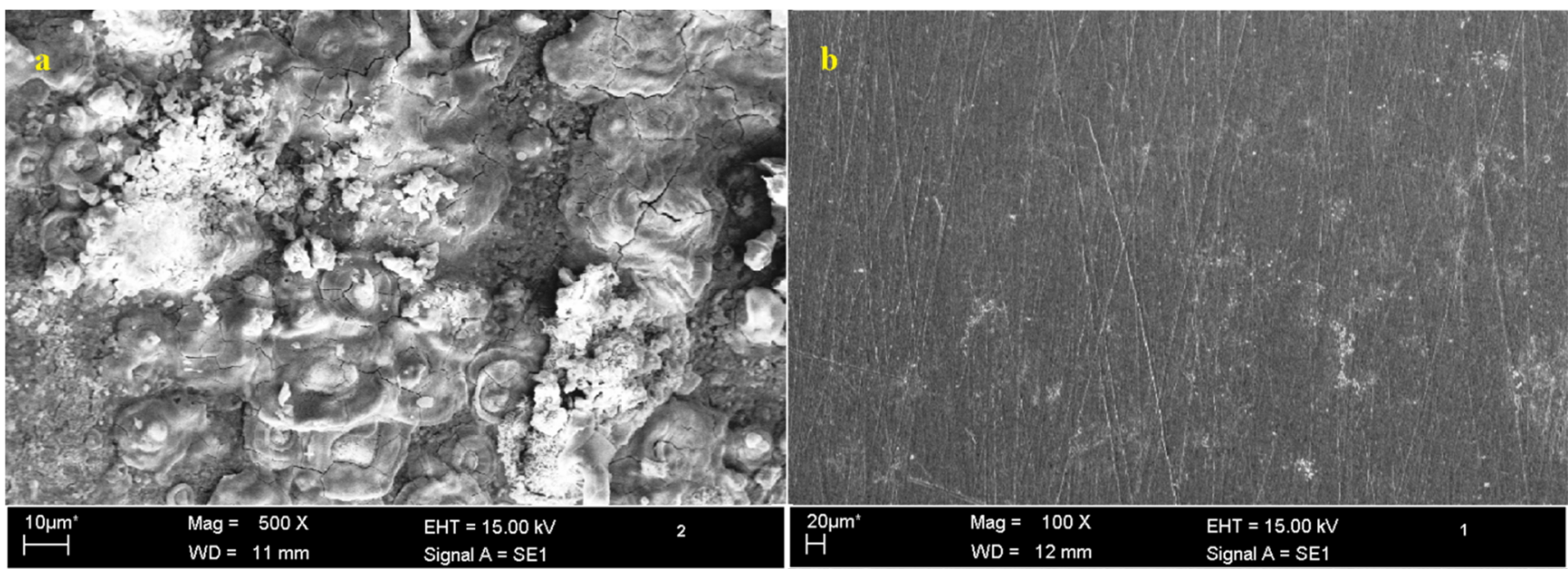

Figure 4. SEM images of the mild steel coupon surfaces: (a) after $5 \mathrm{~h}$ exposure in $1.0 \mathrm{M}$ hydrochloric acid environment without addition of PMO, and (b) after $5 \mathrm{~h}$ exposure in $1.0 \mathrm{M}$ hydrochloric acid environment with addition of $0.005 \mathrm{M}$ of PMO.

Table 1. Calculated parameters for PMO Using DFT method with the $6-31+G(d, p)$ basis set

\begin{tabular}{ll}
\hline Parameter & Value \\
\hline EHOMO $(\mathrm{eV})$ & $-9.056 \mathrm{eV}$ \\
\hline ELUMO $(\mathrm{eV})$ & $-2.707 \mathrm{eV}$ \\
\hline$\Delta \mathrm{E}(\mathrm{eV})$ & 6.349 \\
\hline$\eta$ & 3.174 \\
\hline$\sigma$ & 0.315 \\
\hline$\chi(\mathrm{eV})$ & 5.881 \\
\hline$\Delta \mathrm{N}$ & 1.95 \\
\hline Dipole moment & 2.4533 \\
\hline
\end{tabular}

Figure 5 depicts PMO's optimized geometrical structure. EHOMO and ELUMO, which are the inhibitor's frontier molecular orbital energies, are responsible for their reactivity. Figures 5 show the distributions of the frontier molecule orbital density for PMO. Generally, EHOMO represents the inhibitor's ability to donate electrons. The EHOMO indicates the inhibitor's affinity for offering electrons to suitable acceptor molecules, whereas the ELUMO infers the metal's electron-accepting capacity. The lower the value of LUMO, the more likely the inhibitor will accept electrons from the metal through back-donation. The lower the value of the energy gap (E = ELUMO - EHOMO), the better the molecule's inhibition performance [37]. Other important factors that provide information about molecular stability and reactivity include global hardness ( $\eta$ ) and softness ( $\sigma$ ). A hard molecule has a large energy gap, while a soft molecule has a small one. As a result, the high global hardness $(\eta)$ value and low softness $(\sigma)$ value indicate a higher corrosion inhibition efficiency [38].

PMO molecule (Figure 5), Table 1 demonstrates that the $\Delta \mathrm{E}$ value $(6.349 \mathrm{eV})$ is high, and it is highly correlated with the experimental findings. The softness $(\sigma)$ and a fraction of electrons transferred $(\Delta \mathrm{N})$ values for PMO are both high,

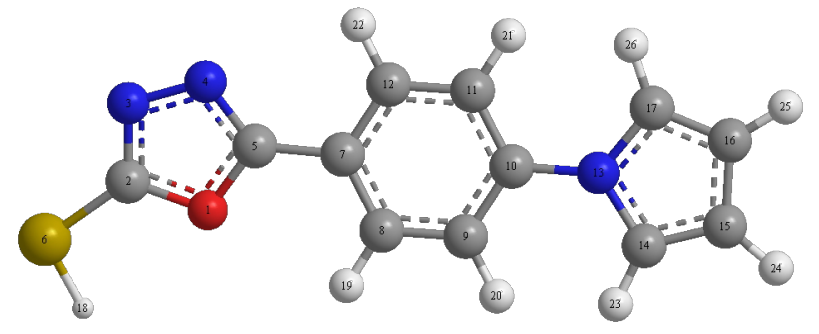

Optimized molecular structure

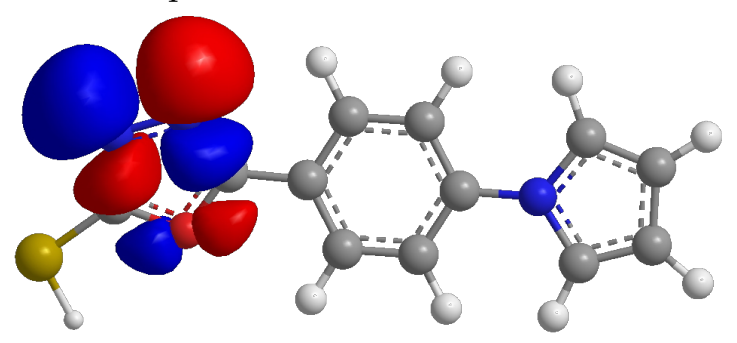

$\mathrm{HOMO}$

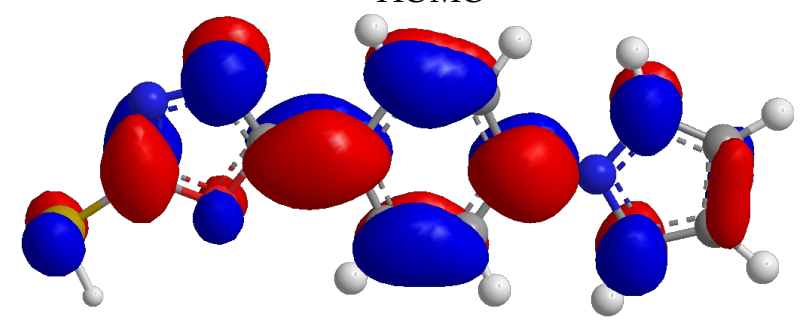

LUMO

Figure 5. The optimized molecular structure, $\mathrm{HOMO}$ and LUMO electron density distribution surfaces of PMO molecule.

while the hardness ( $\eta$ ) value is low, corroborating the experimental findings. PMO's EHOMO has a high value ($9.056 \mathrm{eV})$, which is well supported by the experimental results. The Mulliken charge evaluation is, helpful for calculating inhibitor adsorption sites. The ability to be adsorbed on the mild steel surface increases as the negatively charged heteroatom becomes more negatively charged. Higher negative Mulliken charges are found around the oxygen, nitrogen, and some carbon atoms in PMO molecules, indicating that these atoms are the sites for coordination of 
inhibitor molecules with d-orbital of iron. Table 2 summarizes the Mulliken allegations.

Table 2. Mulliken Charges of Heteroatoms PMO molecules

\begin{tabular}{llllll}
\hline Atom & Atom type & Charges & Atom & Atom type & Charges \\
\hline $\mathrm{O}(1)$ & O Furan & -0.1385 & $\mathrm{C}(10)$ & C Alkene & 0.1567 \\
\hline $\mathrm{C}(2)$ & C Alkene & -0.1318 & $\mathrm{C}(11)$ & C Alkene & -0.1107 \\
\hline $\mathrm{N}(3)$ & N Imine & -0.0913 & $\mathrm{C}(12)$ & C Alkene & 0.0296 \\
\hline $\mathrm{N}(4)$ & N Imine & -0.1246 & $\mathrm{~N}(13)$ & N Pyrrole & -0.199 \\
\hline $\mathrm{C}(5)$ & C Alkene & 0.0876 & $\mathrm{C}(14)$ & C Alkene & -0.0106 \\
\hline $\mathrm{S}(6)$ & S Thiol & 0.2984 & $\mathrm{C}(15)$ & C Alkene & -0.1161 \\
\hline $\mathrm{C}(7)$ & C Alkene & -0.0651 & $\mathrm{C}(16)$ & C Alkene & -0.1172 \\
\hline $\mathrm{C}(8)$ & C Alkene & 0.012 & $\mathrm{C}(17)$ & C Alkene & -0.0066 \\
\hline $\mathrm{C}(9)$ & C Alkene & -0.1109 & $\mathrm{H}(18)$ & H Thiol & -0.0214 \\
\hline
\end{tabular}

\subsection{Mechanism of Corrosion Inhibition}

In most cases, mild steel corrosion in a hydrochloric acid environment is uniform. An attack on the mild steel coupon occurs when it is immersed in a hydrochloric acid environment, with the evolution of hydrogen gas and the formation of iron ions $(\mathrm{Fe} 2+)$. The stages that follow have been used to describe this mechanism [39],

The following equations show how anodic dissolution works:

$$
\begin{aligned}
& \mathrm{Fe}+\mathrm{H}^{+}+e^{-} \rightarrow \mathrm{FeH} \\
& \mathrm{FeH}+\mathrm{FeH}^{+} \rightarrow 2 \mathrm{Fe}+\mathrm{H}_{2} \\
& \left(\mathrm{H}_{2} \mathrm{O}\right)_{a d s} \rightarrow\left(\mathrm{OH}^{-}\right)_{a d s}+\mathrm{H}^{+}(\mathrm{aq}) \\
& \mathrm{Fe}+\left(\mathrm{OH}^{-}\right)_{a d s} \leftrightarrows(\mathrm{Fe}(\mathrm{OH}))_{a d s}+e^{-} \\
& (\mathrm{Fe}(\mathrm{OH}))_{a d s} \rightarrow \mathrm{FeOH}^{-}+e^{-} \\
& \mathrm{FeOH}{ }^{-}+\mathrm{H}^{+} \leftrightarrows \mathrm{Fe}^{+2}+\mathrm{H}_{2} \mathrm{O}
\end{aligned}
$$

In addition, the following equations show how cathodic $\mathrm{H} 2$ evolution occurs:

$$
\begin{aligned}
& \mathrm{Fe}+\mathrm{Fe}_{a d s}^{+} \leftrightarrows\left(\mathrm{FeH}^{+}\right)_{a d s} \\
& \left(\mathrm{FeH}^{+}\right)_{a d s}+e^{-} \leftrightarrows(\mathrm{FeH})_{a d s} \\
& (\mathrm{FeH})_{a d s}+H^{+}+e^{-} \leftrightarrows \mathrm{Fe}+\mathrm{H}_{2}
\end{aligned}
$$

According to the weight loss findings which indicated that the investigated inhibitor molecules act as a significant inhibitor for the mild steel corrosion in a 1.0 M hydrochloric acid environment [40], the adsorption of PMO molecules at the metal/solution interface was used to explain the mechanism of inhibition by the PMO molecules. The adsorption process can be physical, chemical, or a combination of the two adsorption types. The current investigation's thermodynamic parameters indicated that the nature of PMO molecule adsorption on a mild steel surface in a hydrochloric acid environment was primarily chemical and physical. The PMO molecules are excellent ligands that can coordinate with iron and forming coordination complex [41] on the steel surface, forming metal-inhibitor complexes that act as a barrier to protect the mild steel from dissolution, as demonstrated in Figure 6 and the equation below,

$$
\text { Inh }_{\text {ads }}+\mathrm{Fe}^{++} \rightarrow\left[\mathrm{Fe}^{++}-\text {Inh. }\right]_{\text {ads }}
$$

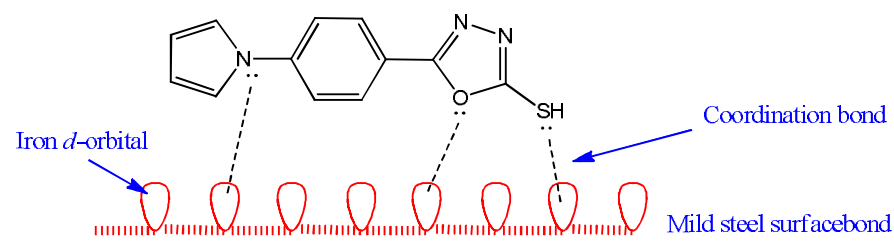

Figure 6. The suggested inhibition mechanism

\section{CONCLUSIONS}

In the current study, a new corrosion inhibitor namely, 5(4-(1H-pyrrol-1-yl)phenyl)-2-mercapto-1,3,4-oxadiazole (PMO), have been synthesized and fully characterized. The inhibition efficiency of PMO molecules versus mild steel corrosion n 1.0 M hydrochloric acid environment at various temperatures (303-333 K) was studied by weight loss techniques and scanning electron microscope. The experimental findings confirmed that $\mathrm{PMO}$ acts as an excellent inhibitor for mild steel corrosion in a $1 \mathrm{M}$ hydrochloric acid environment. The inhibition efficiency was increased with the PMO concentrations, while it reduced with increasing temperature. The SEM photographs revealed that the PMO molecules covered a large area on the mild steel surface. As a result, the high value of inhibition efficiency of the PMO molecules is interpreted as strong organic molecule adsorption on the surface of mild steel. The isotherm adsorption was obeying the Langmuir adsorption. The thermodynamic parameters suggested that the adsorption nature was of the PMO molecules acted as a coexistence of the two adsorption mechanisms (physical and chemical). The inhibition mechanism of the PMO corrosion of mild steel in $\mathrm{HCl}$ and its inhibition by the $\mathrm{PMO}$ was also discussed.

\section{REFERENCES}

1. S.B. Al-Baghdadi, F.G. Hashim, A.Q. Salam, T.K. Abed, T.S. Gaaz, A.A. Al-Amiery, A.A.H. Kadhum, K.S. Reda and W.K. Ahmed, Synthesis and corrosion inhibition application of NATN on mild steel surface in acidic media complemented with DFT studies, Results Phys., 2018, 8, 1178-1184.

https://doi.org/10.1016/j.rinp.2018.02.007

2. K.F. Al-Azawi, I.M. Mohammed, S.B. Al-Baghdadi, T.A. Salman, H.A. Issa, A.A. Al-Amiery, T.S. Gaaz and A.A.H. Kadhum, Experimental and quantum chemical simulations on the corrosion inhibition of mild steel by 3-((5-(3,5-dinitrophenyl)-1,3,4-thiadiazol-2-

yl)imino)indolin-2-one, Results Phys., 2018, 9, 278-283. https://doi.org/10.1016/j.rinp.2018.02.055

3. H.R. Obayes, G.H. Alwan, A.H.MJ. Alobaidy, A.A. AlAmiery, A.A.H. Kadhum and A.B. Mohamad, Quantum chemical assessment of benzimidazole derivatives as corrosion inhibitors, Chem. Cent. J., 2014, 8, no. 21, 1-8. https://doi.org/10.1186/1752-153X-8-21

4. T.A. Salman, D.S. Zinad, S.H. Jaber, M. Shayaa, A. Mahal, M.S. Takriff and A.A. Al-Amiery, Effect of 1,3,4 thiadiazole scafold on the corrosion inhibition of mild steel in acidic medium: an experimental and 
computational study, J. Bio Tribo-Corros., 2019, 5, no. 48, 1-11. https:// doi.org/10.1007/s40735-019-0243-7

5. D.S. Zinad, M. Hanoon, R.D. Salim, S.I. Ibrahim, A.A. AlAmiery, M.S. Takriff and A.A.H. Kadhum, A new synthesized coumarin-derived Schiff base as a corrosion inhibitor of mild steel surface in hcl medium: Gravimetric and dft studies, Int. J. Corros. Scale Inhib., 2020, 9, no. 1, 228-243. https://doi.org/10.17675/23056894-2020-9-1-14

6. D.S. Zinad, Q.A. Jawad, M.A.M. Hussain, A. Mahal, L. Mohamed and A.A. Al-Amiery, Adsorption, temperature and corrosion inhibition studies of a coumarin derivatives corrosion inhibitor for mild steel in acidic medium: Gravimetric and theoretical investigations, Int. J. Corros. Scale Inhib., 2020, 9, no. 1, 534-151. https://doi.org/10.17675/2305-6894-2020-9-1-8

7. M.M. Hanoon, A.M. Resen, A.A. Al-Amiery, A.A.H. Kadhum and M.S. Takriff, Theoretical and Experimental Studies on the Corrosion Inhibition Potentials of 2-((6Methyl-2-Ketoquinolin-3-yl)Methylene) Hydrazinecarbothioamide for Mild Steel in $1 \mathrm{M} \mathrm{HCl}$, Prog. Color Colorants Coat., 2022, 15, 21-33.

8. Lebrini, M.; Bentiss, F.; Vezin, H.; Lagrenee, M. Inhibiting effects of some oxadiazole derivatives on the corrosion of mild steel in perchloric acid solution. Appl. Surf. Sci. 2005, 252, 950-958. https://doi.org/10.1016/j.apsusc.2005.01.160

9. Bouklah, M.; Hammouti, B.; Lagrenee, M.; Bentiss, F. Thermodynamic properties of 2,5-bis(4methoxyphenyl)-1,3,4-oxadiazole as a corrosion inhibitor for mild steel in normal sulfuric acid medium. Corros. Sci. 2006, 48, 2831-2842 https://doi.org/10.1016/j.corsci.2005.08.019

10. S. Junaedi, A.A.H. Kadhum, A. Al-Amiery, A.B. Mohamad and M.S. Takriff, Synthesis and characterization of novel corrosion inhibitor derived from oleic acid: 2-Amino-5- Oleyl-1,3,4-Thiadiazol (AOT), Int. J. Electrochem. Sci., 2012, 7, 3543-3554.

11. A.Y.I. Rubaye, K.S. Rida, A.Q. Salam and A. Al-Amiery, Acetamidocoumarin as a based eco-friendly corrosion inhibitor, Int. J. ChemTech Res., 2016, 9, 39-47.

12. A.A. Al-Amiery, Y.K. Al-Majedy, A.A.H. Kadhum and A.B. Mohamad, New coumarin derivative as an ecofriendly inhibitor of corrosion of mild steel in acid medium, Molecules, 2015, 20, no. 1, 366-383. https://doi.org/10.3390/molecules20010366

13. A.A.H. Kadhum, A.B. Mohamad, L.A. Hammed, A.A. Al-Amiery, N.H. San and A.Y. Musa, Inhibition of mild steel corrosion in hydrochloric acid solution by new coumarin, Mater., 2014, 7, no. 6, 4335-4348. https://doi.org/10.3390/ma7064335

14. A.B. Mohamad, A.A.H. Kadhum, A.A. Al-Amiery, L.C. Ying and A.Y. Musa, Synergistic of a coumarin derivative with potassium iodide on the corrosion inhibition of aluminum alloy in $1.0 \mathrm{M} \mathrm{H} 2 \mathrm{SO} 4$, Met. Mater. Int., 2014, 20, 459-467. https://doi.org/10.1007/s12540-014-3008-3

15. S.S. Al-Taweel, K.W.S. Al-Janabi, H.M. Luaibi, A.A. AlAmiery and T.S. Gaaz, Evaluation and characterization of the symbiotic effect of benzylidene derivative with titanium dioxide nanoparticles on the inhibition of the chemical corrosion of mild steel, Int. J. Corros. Scale Inhib., 2019, 8, no. 4, 1149-1169. https://doi.org/10.17675/2305-6894-2019-8-4-21

16. A. Kadhim, A.A. Al-Amiery, R. Alazawi, M.K.S. AlGhezi and R.H. Abass, Corrosion inhibitors. A review, Int. J. Corros. Scale Inhib., 2021, 10, no. 1, 54-67. https:// doi.org/10.17675/2305-6894-2021-10-1-3

17. S. Junaedi, A. Al-Amiery, A. Kadihum, A.H. Kadhum and A. Mohamad, Inhibition effects of a synthesized novel 4-Aminoantipyrine derivative on the corrosion of mild steel in hydrochloric acid solution together with quantum chemical studies, Int. J. Mol. Sci., 2013, 14, 11915-11928. https://doi.org/10.3390/ijms140611915

18. A. Kadhim,1 A.A. Al-Amiery,2* R. Alazawi,1 M.K.S. AlGhezi2 and R.H. Abass, Corrosion inhibitors. A review, Int. J. Corros. Scale Inhib., 2021, 10, no. 1, 54-67. https:// doi.org/10.17675/2305-6894-2021-10-1-3

19. A.M. Resen, M.M. Hanoon, W.K. Alani, A. Kadhim,3 A.A. Mohammed, T.S. Gaaz, A.A.H. Kadhum, A.A. AlAmiery and M.S. Takriff. Exploration of 8-piperazine-1ylmethylumbelliferone for application as a corrosion inhibitor for mild steel in hydrochloric acid solution. Int. J. Corros. Scale Inhib., 2021, 10, no. 1, 368-387. https:// doi.org/10.17675/2305-6894-2021-10-1-21

20. M. Quraishi, R. Sardar, Aromatic triazoles as corrosion inhibitors for mild steel in acidic environments. Corrosion 2002, 58, 748-755. https:// doi.org/10.5006/1.3277657

21. L. Wang, M. Zhu, F. Yang, C. Gao, Study of a triazole derivative as corrosion inhibitor for mild steel in phosphoric acid solution. Int. J. Corros. 2012, https://doi.org/10.1155/2012/573964

22. P. Liu1, X. Fang, Y. Tang, C. Sun, C. Yao, Electrochemical and quantum chemical studies of 5substituted tetrazoles as corrosion inhibitors for copper in aerated 0.5 M H2SO4 solution. Mater. Sci. Appl. 2011, 2, 1268-1278. https://doi.org/10.4236/msa.2011.29171

23. F. Chaouket, B. Hammouti, S. Kertit, K. Kacemi, Corrosion inhibition of three Fe-B based amorphous alloys in sulphuric acid medium by mercaptophenytetrazole. Bull. Electrochem. 2001, 17, 311- 320 .

24. G. Gomma, M. Wahdan, Effect of copper cation on electrochemical behaviour of steel in presence of imidazole in acid medium. Mater. Chem. Phys. 1997, 47, 176-183. https://doi.org/10.1016/S0254-0584(97)80048$\underline{X}$

25. F. Bentiss, M. Traisnel, H. Vezin, M. Lagrenee, Linear resistance model of the inhibition mechanism of steel in $\mathrm{HCl}$ by triazole and oxadiazole derivatives: structure-activity correlations. Corros. Sci. 2003, 45, 
371-380. https://doi.org/10.1016/S0010-938X(02)00102$\underline{6}$

26. M. Lagrenee, B. Mernari, N. Chaibi, M. Traisnel, H. Vezin, F. Bentiss, Investigation of the inhibitive effect of substituted oxadiazoles on the corrosion of mild steel in $\mathrm{HCl}$ medium. Corros. Sci. 2001, 43, 951-962. https://doi.org/10.1016/S0010-938X(00)00076-7

27. A. Alobaidy, A. Kadhum, S. Al-Baghdadi, A. Al-Amiery, A. Kadhum, E. Yousif et al., Eco-friendly corrosion inhibitor: experimental studies on the corrosion inhibition performance of creatinine for mild steel in $\mathrm{HCl}$ complemented with quantum chemical calculations, Int. J. Electrochem. Sci., 2015, 10, 3961-3972.

28. A. Kadhim, A.K. Al-Okbi, D.M. Jamil, A. Qussay, A.A. Al-Amiery, T.S. Gaas, A.A.H. Kadhum, A.B. Mohamad and M.H. Nassir, Experimental and theoretical studies of benzoxazines corrosion inhibitors, Results Phys., 2017, 7, 4013-4019. https://doi.org/10.1016/j.rinp.2017.10.027

29. H.R. Obayes, A.A. Al-Amiery, G.H. Alwan, T.A. Abdullah, A.A.H. Kadhum and A.B. Mohamad, Sulphonamides as corrosion inhibitor: experimental and DFT studies, J. Mol. Struct., 2017, 1138, 27-34. https://doi.org/10.1016/j.molstruc.2017.02.100

30. Al-Amiery, A.A. Shaker, L. M. Kadhum, A. Takriff, M. Corrosion Inhibition of Mild Steel in Strong Acid Environment by 4-((5,5-dimethyl-3-oxocyclohex-1-en-1yl)amino)benzenesulfonamide. Tribology in industry, 2020, 42, no. 1, 89-101. https://doi.org/10.24874/ti.2020.42.01.09

31. Al-Amiery, A. Salman, T. Alazawi, K. Shaker, L. Kadhum, A. and Takrif, M. Quantum chemical elucidation on corrosion inhibition efficiency of Schiff base: DFT investigations supported by weight loss and SEM techniques. International Journal of Low-Carbon Technologies 2020, 15, 202-209. https://doi.org/10.1093/ijlct/ctz074

32. M. Christov, A. Popova, Adsorption characteristics of corrosion inhibitors from corrosion rate measurements. Corros. Sci. 2004, 46, 1613-1620, https://doi.org/10.1016/j.corsci.2003.10.013

33. S. Shukla, M. Quraishi, Cefotaxime sodium: A new and efficient corrosion inhibitor for mild steel in hydrochloric acid solution. Corros. Sci. 2009, 51, 10071011, https://doi.org/10.1016/i.corsci.2009.02.024

34. M. Behpour, S. Ghoreishi, N. Soltani, M. SalavatiNiasari, M. Hamadanian, A. Gandomi, Electrochemical and theoretical investigation on the corrosion inhibition of mild steel by thiosalicylaldehyde derivatives in hydrochloric acid solution. Corros. Sci. 2008, 50, 21722181, https://doi.org/10.1016/j.corsci.2008.06.020

35. D.M. Jamil, A.K. Al-Okbi, S.B. Al-Baghdadi, A.A. AlAmiery, A. Kadhim and T.S. Gaaz, Experimental and theoretical studies of Schiff bases as corrosion inhibitors, Chem. Cent. J., 2018, 12, no. 7, 1-7. https://doi.org/10.1186/s13065-018-0376-7
36. D. Mahmood, A.K. Al-Okbi, M.M. Hanon, K.S. Rida, A.F. Alkaim, A.A. Al-Amiery, A. Kadhum and A.A.H. Kadhum, Carbethoxythiazole corrosion inhibitor: as an experimentally model and DFT theory, J. Eng. Appl. Sci., 2018, 13, 3952-3959.

37. K. Ansari, M. Quraishi, Experimental and Computational Studies of Naphthyridine Derivatives as Corrosion Inhibitor for N80 Steel in 15\% Hydrochloric Acid. Phys. E 2015, 69, 322- 331, https://doi.org/10.1016/j.physe.2015.01.017

38. L. Olasunkanmi, I. Obot, E. Ebenso, Adsorption and corrosion inhibition properties of $\mathrm{N}-\{\mathrm{n}-[1-\mathrm{R}-5-$ (quinoxalin-6-yl)-4,5-dihydropyrazol- 3 yl] phenyl\} methane sulfon amides on mild steel in $1 \mathrm{M} \mathrm{HCl}$ : experimental and theoretical studies. RSC Adv. 2016, 6, 86782- 86797, https://doi.org/10.1039/C6RA11373G

39. M. M. Hanoon, A. M. Resen, A. A. Al-Amiery, A. A. H. Kadhum, M. S. Takriff, Theoretical and Experimental Studies on the Corrosion Inhibition Potentials of 2-((6Methyl-2-Ketoquinolin-3-yl)Methylene) Hydrazinecarbothioamide for Mild Steel in $1 \mathrm{M} \mathrm{HCl}$. Prog. Color Colorants Coat., 15 (2022), 21-33.

40. A. Rubaye, A. Abdulwahid, S. Al-Baghdadi, A. AlAmiery, A. Kadhum, A. Mohamad, Cheery sticks plant extract as a green corrosion inhibitor complemented with LC-EIS/MS spectroscopy. Int. J. Electrochem. Sci. 2015, 10, 8200- 8209

41. R. D. Salim, N. Betti, M. Hanoon, A. A. Al-Amiery, 2(2,4-Dimethoxybenzylidene)NPhenylhydrazinecarbothioamide as an Efficient Corrosion Inhibitor for Mild Steel in Acidic Environment. Prog. Color Colorants Coat., 15 (2022), 5562. 